\title{
Uniportal Thoracoscopic McKeown Esophagectomy
}

\author{
Zhenghua Zhang $^{1} \cdot$ Baolin Rong ${ }^{1} \cdot$ Mingfa Guo $^{1,2}$ (D) \\ Received: 19 May 2019 / Accepted: 7 February 2020 / Published online: 13 February 2020 \\ (C) Association of Surgeons of India 2020
}

\begin{abstract}
We performed the technique of uniportal thoracoscopic McKeown esophagectomy. The incision was located in the fourth intercostal space on the right axillary midline. The right recurrent laryngeal nerve lymph nodes were dissected. Four hemlocks were applied to clamp the proximal and distal ends of the aortic arch. The esophagus was suspended with purse-string at the level of the azygos vein arch to assist the dissection of the left recurrent laryngeal nerve lymph nodes. A silk thread was drawn out from the incision in order to remove the subcarinal lymph nodes. A thoracic drainage tube was placed at the back of the incision at the end.
\end{abstract}

Keywords Single port · Thoracoscopy $\cdot$ Esophagectomy $\cdot$ Esophageal cancer

\section{Introduction}

After nearly 20 years of improvement and development, thoracoscopic esophagectomy has been carried out in many medical centers around the world. Thoracoscopic esophagectomy had always been done with three holes or four holes. In order to further reduce chest wall trauma and improve postoperative quality of life, we carried out uniportal thoracoscopic McKeown esophagectomy on the basis of mastering the technique of three-hole thoracoscopic esophagectomy. The preliminary results were satisfactory and reported as follows.

\section{Technology}

Single lumen endotracheal intubation combined with occluder was used in anesthesia. The patient was placed on the lateral decubitus position with an anteversion of $30^{\circ}$. A length of about 3-4 cm incision was located in the fourth or fifth intercostal space on the right axillary midline. The thoracoscope was always located at the back of the incision. Thoracoscopic surgery

Mingfa Guo

guomingfa126@126.com

1 Department of Thoracic Surgery, The First Affiliated Hospital of USTC, Division of Life Sciences and Medicine, University of Science and Technology of China, Heifei, Anhui 230001, People's Republic of China

2 Heifei, People's Republic of China was performed according to modularity: (1) The mediastinal pleura above the azygos vein arch was incised to expose the right vagus nerve. We separated retrogradely along the right vagus nerve to reveal the origin of the right recurrent laryngeal nerve. Then the right recurrent laryngeal nerve lymph nodes were dissected (Fig. 1a). After that, we separated the upper esophagus from the spinal side of the esophagus and protected the thoracic duct. (2) We used double joint forceps to separate the azygos vein arch and then used hemlock to clamp the proximal and distal ends of the azygos vein arch (Fig. 1b). (3) The spinal side of the esophagus was separated from the azygos vein arch to the esophageal hiatus, and then the anterior part of the esophagus was free to the bifurcation of the tracheobronchial tract. (4) The esophagus was suspended with purse-string at the level of azygos vein arch (Fig. 2a). The lymph nodes around the left recurrent laryngeal nerve were dissected by esophageal mesangial suspension. (Fig. 2b). The upper esophagus was completely dissociated to the pleura. (5) The esophagus was drawn to the anterior part of the thorax with silk thread, and the silk thread was drawn out from the incision to remove the subcarinal lymph nodes (Fig. 3a). (6) A thoracic drainage tube was placed at the back of the incision (Fig. 3b).

Abdominal and neck operations were the same as those reported before [1].

\section{Results}

Among the 20 patients, there were 15 males and 5 females with an average age of $68.4 \pm 5.3$ years. Postoperative 


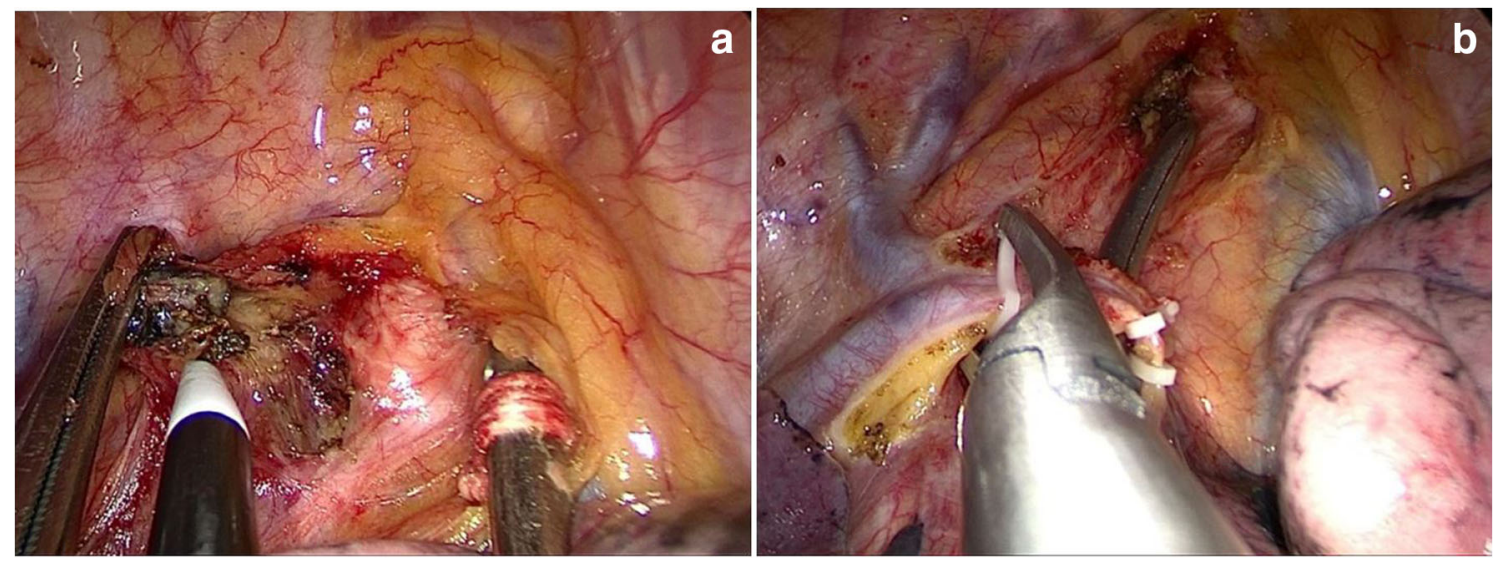

Fig. 1 a Dissection of the right recurrent laryngeal nerve lymph nodes. b The arch of the azygos vein was severed

pathological staging was performed according to the 8th version AJCC (American Joint Committee for Cancer) staging system. There were 4 cases in stage I, 11 cases in stage II, and 5 cases in stage III. The total number of thoracic dissected lymph node was 228 , with an average number of 11.4 dissected lymph node per patient. Two patients had left recurrent laryngeal nerve lymph node metastasis, one had subcarinal lymph node metastasis, and one had middle paraesophageal lymph node metastasis. The average time of thoracic operation was $110.5 \pm 13.9 \mathrm{~min}$ and the average amount of thoracic bleeding was $118 \pm 67.3 \mathrm{ml}$.

Postoperative pulmonary infection occurred in one patient who recovered after 5 days of ventilator-assisted ventilation in ICU (intensive care unit). Postoperative chest incision bleeding occurred in 1 case and was cured by reoperation. There was 1 case of gastric stump fistula that occurred on the fourth day after operation, and was cured by conservative treatment for 26 days after surgery. Postoperative chylothorax occurred in 1 case and was cured by conservative treatment for 3 days. No incision infection and perioperative death occurred.

\section{Discussion}

Because of the high technical difficulty, the progress of uniportal thoracoscopic esophagectomy is relatively slow. In 2016, Lee et al. [2] reported the single-incision laparothoracoscopic minimally invasive esophagectomy to treat esophageal cancer for the first time. Dmitrii [3] and Batirel [4] also reported the experience of uniportal thoracoscopic esophagectomy. Recently, Yuan et al. [5] have also carried out single-port thoracoscopic esophagectomy.

Uniportal thoracoscopic surgery has only one surgical incision, which had relatively less psychological and physiological impact on patients. The incision was located at the fourth or fifth intercostal space on the midaxillary line, where there were few muscles and was not prone to bleeding. The incision at this site caused less muscle and nerve damage than the posterior axillary incision and had less impact on the patient's sensation and movement after surgery. When performing uniportal thoracoscopy, the thoracoscopic lens was directly aligned to the surgical field of view. As a result, the depth of vision made it easier for surgeons to determine the operating
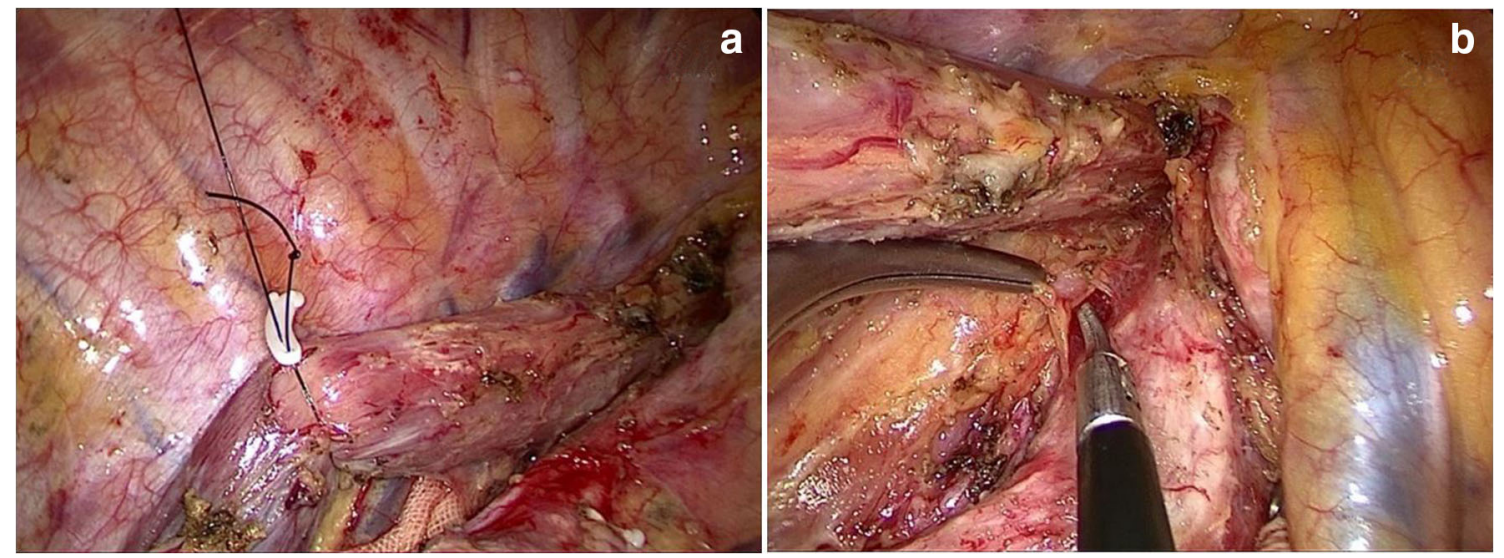

Fig. 2 a The esophagus was drawn with a purse-string at the level of the azygos arch. b Removal of the left recurrent laryngeal nerve lymph nodes 

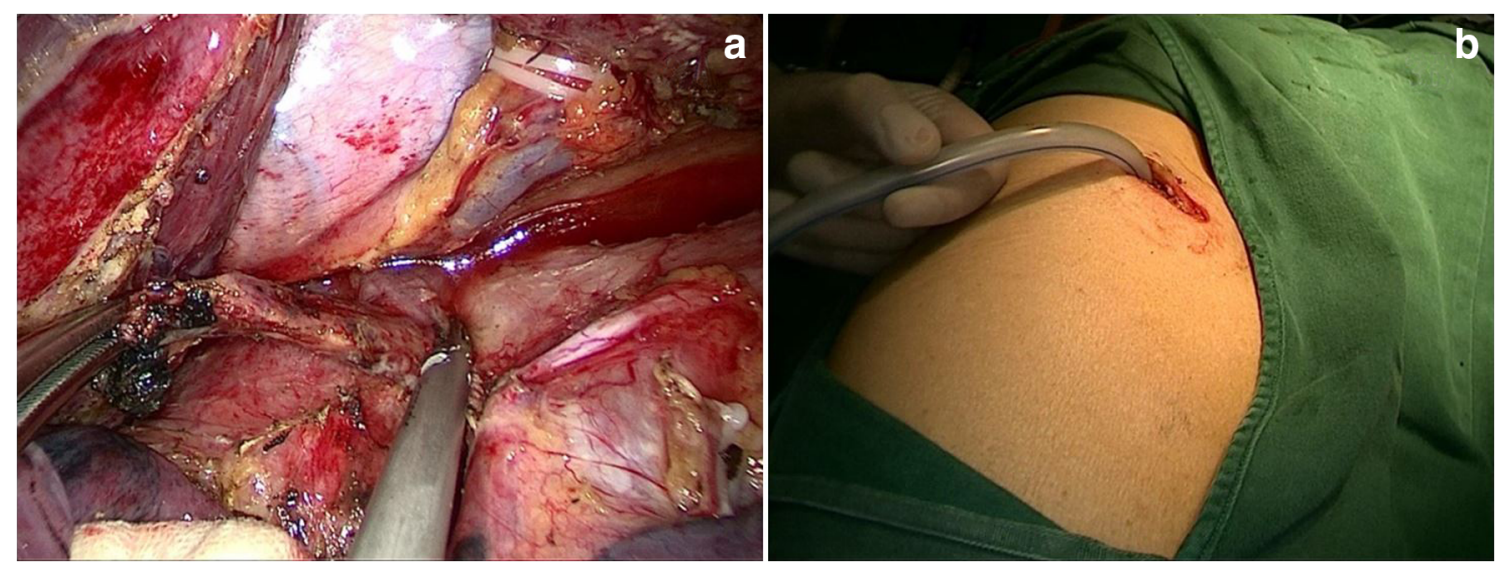

Fig. 3 a Removal of subcarinal lymph nodes. b The thoracic incision and drainage tube

distance, which made the surgical field of vision and mode of operation closer to the traditional thoracotomy. The results of this study showed that the average time of thoracic operation was $110.5 \pm 13.9 \mathrm{~min}$, and the average amount of thoracic bleeding was $118 \pm 67.3 \mathrm{ml}$ respectively, indicating the safety of single-port thoracoscopic esophagectomy.

Our initial experience was as follows. Firstly, the esophagus and lymph nodes were dissected according to the fixed pattern, so that the operation process was more smoothly and the surgeon and assistant could cooperate more tacitly. Secondly, we applied various methods to free the esophagus during operation. For example, after complete dissociation of the middle and lower esophagus, a purse-string was inserted through the fourth or fifth intercostal space on the medial margin of scapula to tract the esophagus and so as to facilitate the dissociation of the upper esophagus and the clearance of the left recurrent laryngeal nerve lymph nodes. When the tumor volume was relatively large, we used ribbon gauze to pull the normal esophagus at the upper and lower ends of the tumor, or we cut the esophagus with a cutting suture device to facilitate exposure and dissociation. Thirdly, the traditional instruments were improved during the operation. We used cotton thread to knot at the end of the elbow suction apparatus, which increased the friction between the suction apparatus and the esophageal tissues and facilitated the use of the suction apparatus to separate the esophagus. Fourthly, it was better to use a single-lumen tube and occluder in anesthesia, because the double-lumen tracheal intubation is thicker in diameter and harder in texture, which is not conducive to dissect the left recurrent laryngeal nerve lymph node.

However, there are many limitations in single-port thoracoscopic esophagectomy. Firstly, it is more difficult to expose and free esophagus and mediastinal lymph nodes by single-hole thoracoscopic esophagectomy than by multi-hole thoracoscopic esophagectomy. Multiple instruments can easily interfere with each other in one incision. Secondly, the thoracic esophagus is located in the posterior mediastinum and begins at the top of the pleura and ends in the hiatus, which increases the difficulty of the operation. Thirdly, single-hole thoracoscopic esophagectomy may require a longer learning curve, which needs stricter risk prevention and control measures. Fourthly, single-hole thoracoscopic surgery should not be forced to perform for large tumors involving the adventitia of the esophagus. Therefore, we suggested that the early cases should be selected by uniportal thoracoscopic esophagectomy. Uniportal thoracoscopic esophagectomy is a more thorough and minimally invasive esophageal surgery. We believed that, with the development of endoscopic techniques and the accumulation of experience, more patients are expected to benefit from it.

\section{Compliance with Ethical Standards}

Conflict of Interest The authors declare that they have no conflict of interest.

\section{References}

1. Luketich JD, Alvelo-Rivera M, Buenaventura PO et al (2003) Minimally invasive esophagectomy: outcomes in 222 patients. Ann Surg 238(4):486-494 discussion 494-5

2. Lee JM, Yang SM, Yang PW, Huang PM (2016) Single-incision laparo-thoracoscopic minimally invasive oesophagectomy to treat oesophageal cancer. Eur J Cardiothorac Surg 49(Suppl 1):i59-i63

3. Dmitrii S, Pavel K (2017) Uniportal video-assisted thoracic surgery esophagectomy. Thorac Surg Clin 27(4):407-415

4. Batirel HF (2017) Uniportal video-assisted thoracic surgery for esophageal cancer. J Vis Surg 3:156

5. Hu W, Yuan Y, Chen L (2019) Single-port thoracoscopic minimally invasive esophagectomy for esophageal cancer. World J Surg 43(2): $567-570$

Publisher's Note Springer Nature remains neutral with regard to jurisdictional claims in published maps and institutional affiliations. 